\title{
Revisiting DPPH (2,2-diphenyl-1-picrylhydrazyl) assay as a useful tool in antioxidant evaluation: a new IC100 concept to address its limitations
}

\author{
JuDong Yeo and Fereidoon Shahidi*
}

\begin{abstract}
Department of Biochemistry, Memorial University of Newfoundland, St. John's, NL, Canada A1B 3X9
${ }^{*}$ Corresponding author: Fereidoon Shahidi, Department of Biochemistry, Memorial University of Newfoundland, St. John's, NL, Canada A1B 3X9. Tel: +1 709864 8552; E-mail: fshahidi@mun.ca

DOI: $10.31665 /$ JFB.2019.7196

Received: September 06, 2019; Revised received \& accepted: September 28, 2019

Citation: Yeo, J.D., and Shahidi, F. (2019). Revisiting DPPH (2,2-diphenyl-1-picrylhydrazyl) assay as a useful tool in antioxidant evaluation: a new IC100 concept to address its limitations. J. Food Bioact. 7: 36-42.
\end{abstract}

\begin{abstract}
The DPPH (2,2-diphenyl-1-picrylhydrazyl) assay has been widely used in antioxidant evaluation. However, it suffers from certain limitations that are addressed in this contribution. The limitations discussed in this work were the ratio of DPPH radicals to antioxidants and the presence of pigments in the reaction medium which interferes with absorbance readings. To do so, we used eight different concentrations of DPPH solution. The modified DPPH assay proposes a new concept, $\mathrm{IC}_{100}$, that is defined as the amount of DPPH radical required to oxidize all antioxidants present in the reaction medium. The modified DPPH assay does not suffer from an underestimation of antioxidant activity found in the original DPPH procedure due to the decrease in the ratio of DPPH radicals to antioxidants. Moreover, the modified method was not influenced by interference from coexisting pigments in the measurement of radical scavenging potential of extracts. To the best of our knowledge, this is the first attempt to effectively resolve the above-mentioned limitations of the DPPH assay.
\end{abstract}

Keywords: $\mathrm{IC}_{100}$; DPPH; Radical scavenging ability; Stoichiometric factor; Antioxidant capacity.

\section{Introduction}

Free radicals with one or more unpaired electron are naturally occurring in biological and food systems; these include hydroxyl $(\mathrm{HO} \bullet$ ), lipid alkyl $(\mathrm{L} \bullet)$, alkoxyl $(\mathrm{LO} \bullet)$, and lipid peroxyl $(\mathrm{LOO} \bullet)$ radicals (Pryor, 1986). As evidenced in their short lifetime of $10^{-9}$ to 10 seconds, they are highly reactive and unstable entities. This aggressive property of free radicals causes damage to the cells, leading to many chronic diseases such as atherosclerosis, ageing, cancer and several other ailments (Aruoma, 1998).

Recently, a large body of research has been conducted in order to explore natural radical scavengers which can suppress the aggressive tendency of free radicals. In this connection, plant-based foods provide excellent sources of free radical scavengers such as phenolic compounds. These include phenolic acids such as gallic acid, protocatechuic acid, $p$-coumaric acid, and ferulic acid as well as flavonoids including quercetin, kaempferol, and catechins that are found in seeds, cereals, legumes, fruits, and vegetables (Shahidi and Yeo, 2016; Shahidi and Peng, 2018). Natural antioxidants also exhibit a potent free radical scavenging potential by displaying excellent electron or hydrogen atom donating ability to free radicals (Litwinienko and Ingold, 2003; Foti and Ruberto, 2000). In this contribution, the traditional 2,2-diphenyl-1-picrylhydrazyl (DPPH) assay that is popularly used in the evaluation of free radical scavenging potential of antioxidants is revisited to address its shortcomings.

In 1958, Blois reported the oxidation of antioxidants such as cysteine, glutathione, ascorbic acid, tocopherol, as well as polyhydroxy and aromatic compounds by DPPH radical. Since then DPPH radical has been widely used as a popular reagent for the determination of electron or hydrogen atom donating potential of antioxidants in food and in polymer chemistry research (Blois, 1958; Fargere et al., 1995). In the UV-visible spectrum, DPPH radical has three main 


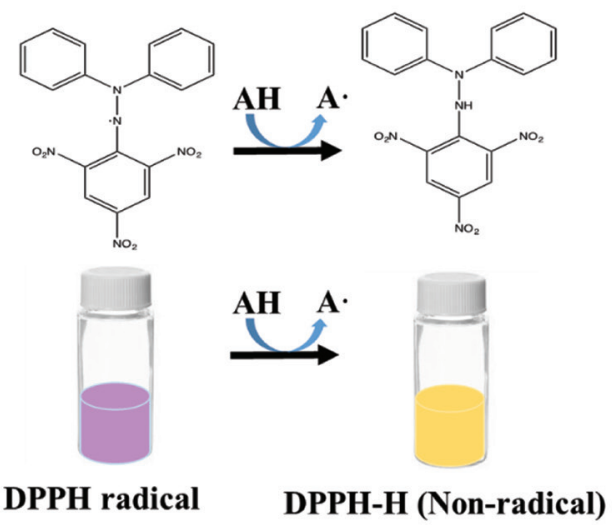

b

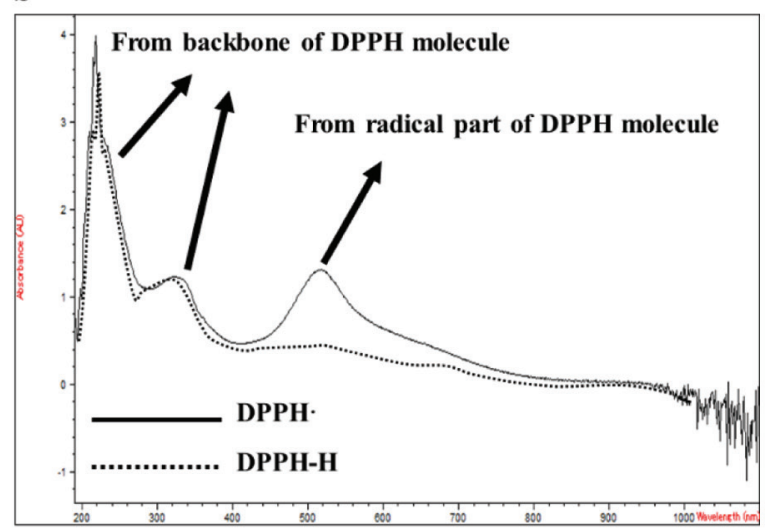

Figure 1. The changes of chemical structure (a), color (a), and the absorption spectrum of DPPH radical after reduction by an antioxidant.

peaks in which two peaks below $400 \mathrm{~nm}$ are derived mainly from the DPPH. Another peak around $517 \mathrm{~nm}$ is due to the resonance of the radical, that changes upon the reduction of DPPH radical by antioxidants. However, peaks at 220 and $324 \mathrm{~nm}$ are not affected to any great extent and with only a slight blue shift of the $324 \mathrm{~nm}$ peak due to the stabilization of the DPPH molecule after reduction (Figure 1). Thus, the absorbance at $517 \mathrm{~nm}$ is monitored in the DPPH assay and the reason for routinely using DPPH assay for the determination of hydrogen atom or electron donating capacity of bioactive molecules.

Meanwhile, there are certain shortcomings in the DPPH assay which may adversely affect the accuracy of the results; firstly, the ratio of DPPH radical to antioxidant in the reaction medium affects the electron transfer or hydrogen atom donating potential of antioxidants. The lack of standardization in the ratio of DPPH to antioxidant makes it hard to compare the results with those in the existing literature. This limitation has already been noted in the published work. Deng et al. (2011) argued that " $\mathrm{IC}_{50}$ value changes depending on the final concentration of antioxidant in the DPPH solution". For example, $\mathrm{IC}_{50}$ of 10 antioxidants varied about 2-3 times when three different DPPH concentrations were used. Other studies have also pointed out to similar limitations of the DPPH assay (Scherer and Godoy, 2009; Sun and Ho, 2005; van den Berg et al., 1999). Furthermore, the pigments which may coexist in the extracts could interfere with the determinations by absorbing in the same wavelength range of DPPH radicals (around $517 \mathrm{~nm}$ ), leading to underestimation of the final results. Thus, this study was designed to propose a possible solution to address concerns about the accuracy and reproducibility of the original DPPH method which has been widely used to measure antioxidant capacity of molecules since it was first considered by Blois (1958). In a recent publication, Yeo and Shahidi (2019) suggested the use of electron paramagnetic resonance (EPR) for evaluation of the DPPH scavenging of colored extracts to eliminate interference The core hypothesis of the present study was to adopt a wide range of DPPH concentrations to circumvent the ratio of DPPH t to antioxidant and pigment interference in the original DPPH assay and to device a new index which can express the results so obtained in a precise manner.

\section{Materials and methods}

\subsection{Materials}

The 2,2-diphenyl-1-picrylhydrazyl (DPPH), butylated hydroxy- toluene (BHT), $\alpha$-tocopherol, ascorbyl palmitate, and catechin were purchased from Sigma-Aldrich Canada Ltd. (Oakville, ON, Canada). Methanol and ethanol were bought from Fisher Scientific Co. (Nepean, ON, Canada). Blackberry, raspberry, bell pepper and beet were bought from a local market in St. John's, NL, Canada. All other chemicals and reagents were procured from commercial sources and were used without any further purification.

\subsection{The original DPPH radical scavenging assay}

The original DPPH radical scavenging assay of representative antioxidants and food-extracts was performed according to the procedure described by Madhujith and Shahidi (2006) with minor modifications. The DPPH solution $(0.2 \mathrm{mM}, 1.9 \mathrm{~mL})$ in methanol was allowed to react with $0.1 \mathrm{~mL}$ of each sample $(1 \mathrm{~g}$ of dried weight $/ 30 \mathrm{~mL}$ ) and kept in the dark for $30 \mathrm{~min}$. DPPH radical scavenging activity was measured by reading the absorbance at $517 \mathrm{~nm}$.

\subsection{Modified DPPH assay}

The modified DPPH method was employed by using a wide range of DPPH concentrations as graphically shown in Figure 2. Briefly, $0.1 \mathrm{~mL}$ of antioxidant $(0.25,0.50$, and $1.00 \mathrm{mM})$ or extract $(25,50$, and $100 \mathrm{mg} / \mathrm{mL}$ ) was mixed with $1.9 \mathrm{~mL}$ of different concentrations of DPPH solution $(0.1-0.8 \mathrm{mM})$, followed by $30 \mathrm{~min}$ reaction time in the dark. The absorbance was read using a diode array spectrophotometer (Agilent, Palo Alto, CA, USA) at $517 \mathrm{~nm}$. The intensity of the remaining purple-colored DPPH radicals increased gradually depending on the concentration of the DPPH solution. This allowed determination of the specific point that the absorbance began to increase, which we refer to as the "Inhibitory Concentration 100 or $\left(\mathrm{IC}_{100}\right)$ ". The $\mathrm{IC}_{100}$ indicates "the concentration of DPPH radical needed to abstract $100 \%$ of the available hydrogen atoms of the antioxidant present in the reaction medium". This new concept was used again to calculate the stoichiometry factor of the pure antioxidant compound or extract.

\subsection{Determination of the $I C_{100}$, the stoichiometry factor, and a new unit of scavenged DPPH radical in $\mathrm{mg} / \mathrm{g}$ of sample}

The determination of $\mathrm{IC}_{100}$ was carried out as summarized in Fig- 

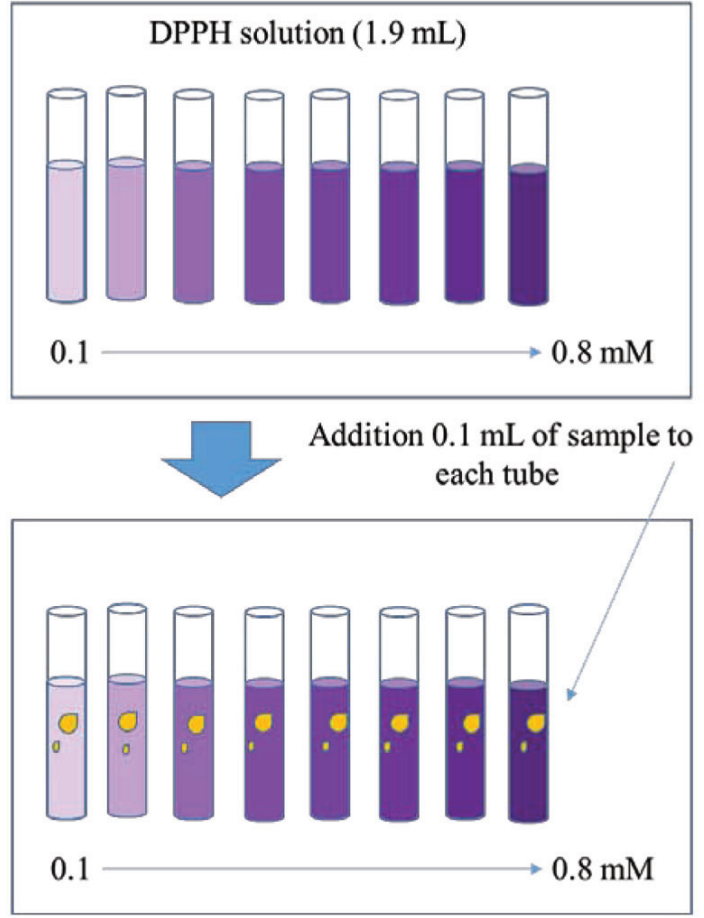
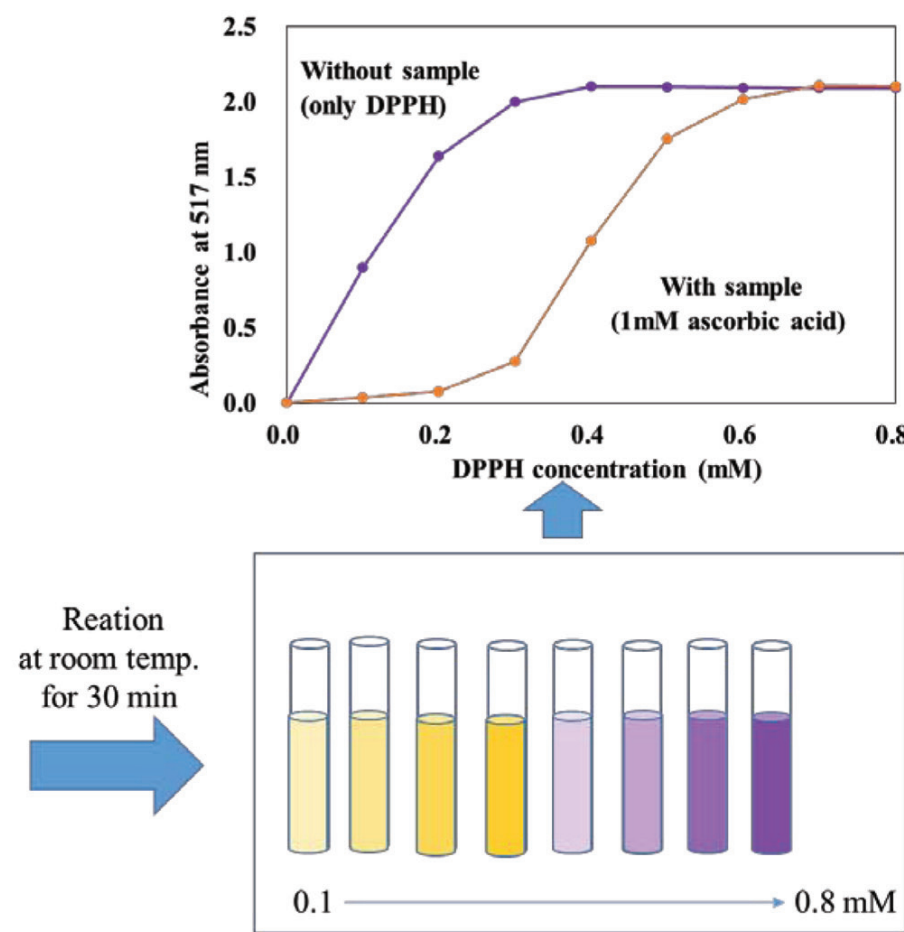

Figure 2. Procedure of the modified DPPH procedure.

ure 3. The $\mathrm{IC}_{100}$ indicates the specific concentration of the DPPH solution at which absorbance begins to increase after reaction with an antioxidant, defined as the $\mathrm{IC}_{100}$. For the determination of $\mathrm{IC}_{100}$, two equations are proposed using the pattern in the graph before and after the $\mathrm{IC}_{100}$ followed by calculating the exact $\mathrm{IC}_{100}$, as explained in Figure 3. The $\mathrm{IC}_{100}$ was further used to determine the stoichiometry factors of the pure representative antioxidants. Stoichiometry factor was calculated by dividing $\mathrm{IC}_{100}$ by the concentration of antioxidant used to obtain the number of hydrogen atoms or electrons to scavenge one molecule of DPPH radical. The $\mathrm{IC}_{100}$ so obtained was also converted into the scavenged DPPH radical in $\mathrm{mg} / \mathrm{g}$ of dry weight (DW) of defatted samples for the extracts using the following equation.

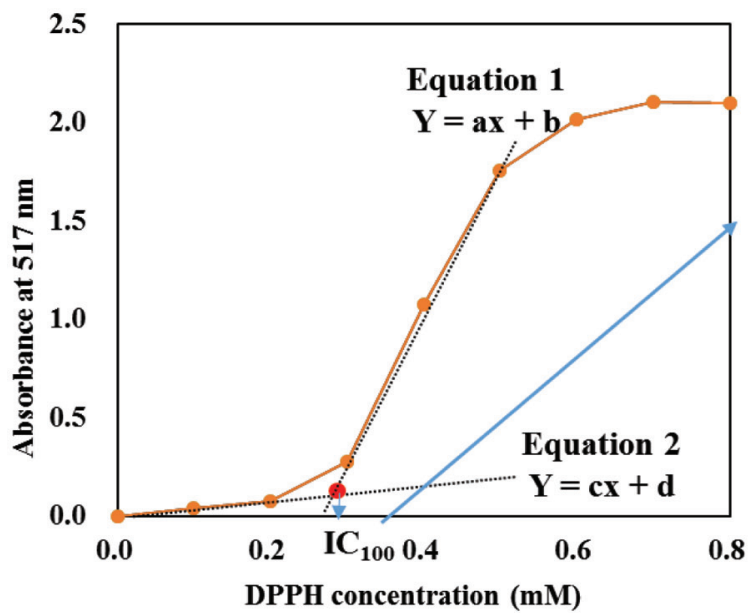

Scavenged DPPH $\mathrm{mg} / \mathrm{g}$ of DW

$$
=\frac{\mathrm{IC}_{100}(\mathrm{mM}) \times 394(\mathrm{MW} \text { of } \mathrm{DPPH})}{\text { Concentration of extract }(\mathrm{mg} / \mathrm{Ml})}
$$

\subsection{Extraction of blackberry, raspberry, bell pepper, and beet}

The extraction was carried out as described by Yeo and Shahidi (2019) with slight modification. The fresh blackberry, raspberry, bell pepper, and beet were finely ground using a blender, followed by lyophilization. One gram of dried sample was subsequently mixed with $10 \mathrm{~mL}$ of $70 \%$ methanol for $20 \mathrm{~min}$ at ambient tem-

IC $_{\mathbf{1 0 0}}$ indicate the required DPPH concentration to oxidized all antioxidants used

Calculation of $\mathrm{IC}_{100}$ using equation1. and 2. $\mathrm{ax}+\mathrm{b}=\mathrm{cx}+\mathrm{d}$

$(a-c) x=(d-b)$

$\mathrm{x}=\mathrm{d}-\mathrm{b} /(\mathrm{a}-\mathrm{c})$

\section{Calculation of stoichiometry factor}

$=\mathbf{I C}_{\mathbf{1 0 0}}(\mathrm{mM}) /$ concentration of antioxidant $(\mathrm{mM})$

Figure 3. Determination of $\mathrm{IC}_{100}$ by using two equations having a different pattern in the measurement of DPPH radical scavenging ability. 
Table 1. DPPH radical scavenging ability of representative antioxidants using the original DPPH assay

\begin{tabular}{|c|c|c|c|c|}
\hline & $\begin{array}{l}\text { Concentration } \\
\text { (mM) }\end{array}$ & $\begin{array}{l}\text { Ratio } \\
\text { (DPPH/Antioxidant) }\end{array}$ & $\begin{array}{l}\text { Scavenged DPPH } \\
\text { (\%) }\end{array}$ & $\begin{array}{l}\text { Stoichiometric factor } \\
\text { (n, DPPH/Antioxidant) }\end{array}$ \\
\hline \multirow[t]{5}{*}{$\alpha$-Tocopherol } & 1.000 & $2 / 1$ & $65.7 \pm 0.5$ & 1.3 \\
\hline & 0.500 & $4 / 1$ & $51.8 \pm 1.1$ & 2.1 \\
\hline & 0.250 & $8 / 1$ & $27.5 \pm 1.3$ & 2.2 \\
\hline & 0.125 & $16 / 1$ & $17.1 \pm 0.8$ & 2.7 \\
\hline & 0.063 & $32 / 1$ & $10.1 \pm 0.5$ & 3.2 \\
\hline \multirow[t]{5}{*}{ Ascorbyl-6-palmitate } & 1.000 & $2 / 1$ & $31.1 \pm 2.1$ & 0.6 \\
\hline & 0.500 & $4 / 1$ & $17.5 \pm 0.6$ & 0.7 \\
\hline & 0.250 & $8 / 1$ & $10.4 \pm 1.5$ & 0.8 \\
\hline & 0.125 & $16 / 1$ & $6.7 \pm 0.8$ & 1.1 \\
\hline & 0.063 & $32 / 1$ & $4.1 \pm 0.1$ & 1.3 \\
\hline \multirow[t]{5}{*}{ BHT } & 1.000 & $2 / 1$ & $62.7 \pm 0.6$ & 1.3 \\
\hline & 0.500 & $4 / 1$ & $46.9 \pm 1.4$ & 1.9 \\
\hline & 0.250 & $8 / 1$ & $30.1 \pm 0.3$ & 2.4 \\
\hline & 0.125 & $16 / 1$ & $17.7 \pm 0.1$ & 2.8 \\
\hline & 0.063 & $32 / 1$ & $11.4 \pm 0.3$ & 3.6 \\
\hline \multirow[t]{5}{*}{ Catechin } & 1.000 & $2 / 1$ & $67.9 \pm 2.1$ & 1.4 \\
\hline & 0.500 & $4 / 1$ & $68.1 \pm 1.7$ & 2.7 \\
\hline & 0.250 & $8 / 1$ & $61.2 \pm 1.5$ & 4.9 \\
\hline & 0.125 & $16 / 1$ & $41.6 \pm 1.0$ & 6.7 \\
\hline & 0.063 & $32 / 1$ & $26.9 \pm 0.3$ & 8.6 \\
\hline
\end{tabular}

perature, and this step was repeated two more times. The extracts were then filtered through a Whatman No. 1 filter paper in order to remove any remaining solid particles in the samples. The extracts were then kept at $-40 \mathrm{C}$ for further analysis.

\section{Results and discussion}

\subsection{Limitation of the DPPH assay as reflected in the ratio of $D P P H$ radicals to antioxidants}

The results in the DPPH assay are affected by the ratio of DPPH radical to the antioxidant in the reaction medium as summarized in Table 1. The ratios of DPPH - to antioxidant selected were $2 / 1,4 / 1$, $8 / 1,16 / 1$, and 32/1. For comparison of DPPH radical scavenging activity (DRSA), the results were expressed as the stoichiometry factor which is defined as the number of hydrogen atoms or electrons that the antioxidant molecule may donate to the DPPH radical. As expected, the stoichiometry factors of antioxidants were greatly affected by the ratios of DPPH - to antioxidant, namely the stoichiometry factors of $\alpha$-tocopherol, ascorbyl-6-palmitate, butylated hydroxytoluene (BHT) and catechin were significantly increased approximately 2-6 times with the increase of the ratio from $2 / 1$ to $32 / 1$. This indicates that the number of hydrogen atoms or electrons that the antioxidants can donate varies depending on the ratio of DPPH radical to antioxidant in the reaction medium. Several studies have shown the discrepancy of DRSA of antioxidants at the different ratios of DPPH radical to antioxidant. For example, a large variation existed among the reports in the determination of $\mathrm{IC}_{50}$ of ascorbic acid, being 5.8, 50.0, 62.4, and $110.7 \mu \mathrm{g} / \mathrm{mL}$ (Kanimozhi and Prasad, 2009; Charrier et al., 2006; Shirwaikar et al., 2006; Ricci et al., 2005). This observation was also made by Deng et al. (2011), Scherer and Godoy (2009), Sun and Ho (2005) as well as van den Berg et al. (1999). The reason for the discrepancy might be due to the reversibility of the reaction. According to Bondet et al. (1997) and Huang et al. (2005), some of DPPH-H which is formed after receiving a hydrogen atom from the antioxidants may be converted back to DPPH radical due to the chemical equilibrium of the reaction medium. Therefore, at a low concentration of antioxidant in the reaction medium, a low amount of DPPH$\mathrm{H}$ will be produced, followed by a low amount of re-conversion of DPPH-H to DPPH radical. Conversely, at a high concentration of antioxidant a high amount of DPPH-H is re-converted to the DPPH radical, hence this may lead to the more underestimation of the DPPH radical scavenging potential of antioxidant. Thus, to address this limitation of the DPPH assay a viable solution that could circumvent the underestimation of DPPH radical scavenging ability of antioxidants is deemed necessary.

\subsection{Applying the modified DPPH assay to representative antioxidants}

As mentioned earlier, one needs to overcome the reversibility of DPPH-H back to the DPPH radical in the reaction medium to address the ratio issue. For that, we employed a wide range of DPPH concentrations ranging from 0.1 to $0.8 \mathrm{mM}$ to avoid the reversibil- 
Table 2. The $\mathrm{IC}_{100}$ and stoichiometric factors of representative antioxidants using the modified DPPH method

\begin{tabular}{|c|c|c|c|}
\hline & Concentration (mM) & $\mathrm{IC}_{100}(\mathrm{mM})$ & $\begin{array}{l}\text { Stoichiometric factor } \\
\text { (n, DPPH/Antioxidant) }\end{array}$ \\
\hline \multirow[t]{3}{*}{$\alpha$-Tocopherol } & 1.00 & 0.124 & 2.488 \\
\hline & 0.50 & 0.060 & 2.389 \\
\hline & 0.25 & 0.025 & 1.985 \\
\hline \multirow[t]{3}{*}{ Ascorbyl 6-palmitate } & 1.00 & 0.133 & 2.655 \\
\hline & 0.50 & 0.060 & 2.396 \\
\hline & 0.25 & ND & ND \\
\hline \multirow[t]{3}{*}{$\mathrm{BHT}$} & 1.00 & 0.011 & 0.221 \\
\hline & 0.50 & 0.007 & 0.265 \\
\hline & 0.25 & ND & ND \\
\hline \multirow[t]{3}{*}{ Catechin } & 1.00 & 0.198 & 3.963 \\
\hline & 0.50 & 0.103 & 4.106 \\
\hline & 0.25 & 0.045 & 3.637 \\
\hline
\end{tabular}

ity. Four representative antioxidants, $\alpha$-tocopherol, ascorbyl-6-palmitate, BHT, and catechin, were tested to evaluate the suitability of the modified DPPH assay, and the following results were calculated into $\mathrm{IC}_{100}$ and the stoichiometry factor. The stoichiometry factor is a unique property of individual antioxidants that should provide the same value, regardless of the different ratios of the DPPH radical to the antioxidant; thus, the stoichiometry factor can serve as an appropriate parameter to test the suitability of the modified method (Table 2). Unlike the original DPPH assay given in Table 1, the stoichiometry factor by the modified method did not show any marked increasing or decreasing trend due to the changes in the ratio of DPPH radical to the antioxidant. Thus, the ratio of DPPH to antioxidant is no longer an issue in the modified method, possibly due to the minimization of the reversibility of DPPH-H back to the DPPH radical by adopting a wide range of DPPH concentrations.

Meanwhile, the modified method is considered as a more appropriate way to yield stoichiometry factor of antioxidants compared to the original DPPH assay. According to the literature, $\mathrm{IC}_{50}$ obtained by the original method suffers from serious errors itself; that is, the $\mathrm{IC}_{50}$ showed marked variations depending on the ratio of DPPH radical to antioxidants adopted, leading to the erroneous stoichiometry factor (Scherer and Godoy, 2009; Sun and Ho, 2005; van den Berg et al., 1999). However, the stoichiometry factor yielded by the modified method considering the $\mathrm{IC}_{100}$ displayed a constant value of stoichiometry factor at different ratios of DPPH radical to the antioxidant.

In addition, the stoichiometry factor provides a valuable opportunity to explore the mechanism of hydrogen atom or electron donating potential of antioxidants. For example, stoichiometry factors of $\alpha$-tocopherol, ascorbyl-6-palmitate, BHT, and catechin, determined by modified method, were $2.3,2.5,0.2$, and 3.9, respectively. Given the corresponding number of hydroxyl groups in their chemical structures as $1,3,1$, and 5, respectively, a high dependency between stoichiometry factors by the modified method and the number of hydroxyl groups is expected. Shi and Niki (1998) reported that kaempferol showed the experimental stoichiometric numbers of 1.9 , which is lower than the number of hydroxyl groups (4) of kaempferol. Cheng et al. (1998) noted that butein and ascorbic acid, which have four hydroxyl groups, also showed lower stoichiometry factors than it is expected by displaying 1.4 and 0.7 , respectively. Thus, a discrepancy between stoichiometry factor and number of hydroxyl groups was found in the experimental data depending on the reaction environment.

In the present study, the correlation coefficient between stoichiometry factor calculated by the modified method and number of hydroxyl groups was 0.98 , showing a high value with the exception of $\alpha$-tocopherol. Once $\alpha$-tocopherol is included, the correlation coefficient dropped to 0.70 , which means that the number of hydroxyl groups of $\alpha$-tocopherol (only 1) does not match with the number of hydrogen atom or electron it can donate; in other words, $\alpha$-tocopherol donates one more hydrogen atom or electron to the DPPH radical. The difference between the number of hydroxyl groups of $\alpha$-tocopherol and the number of hydrogen atoms or electrons it can donate is related to the hydrogen atom or electron donating mechanism; in this $\alpha$-tocopherol becomes phenoxyl radical after donating a hydrogen atom, subsequently it can donate one more electron, leading to the formation of $\alpha$-tocopheryl quinone (Decker, 2002). Thus, $\alpha$-tocopherol can donate one more electron or hydrogen atom in the process, which may be the reason for the observed disagreement. Therefore, determination of the stoichiometry factor based on the modified DPPH assay not only can improve the accuracy in the evaluation of hydrogen atom or electron donating ability of antioxidant, but also allows a critical discussion about the possible hydrogen atom or electron donating mechanism of antioxidant by comparing the stoichiometry factor and the number of hydroxyl groups present.

Up to the present day, many attempts for the expression of DPPH radical scavenging potential of antioxidants have been made, namely $\mathrm{IC}_{50}, \mathrm{EC}_{50}$, and the inhibition of the $\mathrm{DPPH}$ radical in percent (I\%) (Ani et al., 2006; Elzaawely et al., 2007; Guerrero et al., 2006). Moreover, Scherer and Godoy (2009) proposed a new formula referred to as antioxidant activity index (AAI) and Deng et al. (2011) suggested a novel index such as antioxidant activity unit (AAU) by considering the volume ratio of the sample to DPPH solution and the molecular weight of antioxidants as well. All such approaches for the expression of yielded results have successfully been adopted. However, since they used only one concentration of DPPH solution (one ratio of DPPH radical/antioxidants) in the measurement, the expressed values cannot be free from the ratio 
Table 3. The calculation of $\mathrm{IC}_{100}$ and scavenged DPPH radical by different food extracts

\begin{tabular}{llll}
\hline & Concentration $(\mathbf{m g} / \mathbf{m L})$ & $I_{100}(\mathbf{m M})$ & Scavenged DPPH $\mathbf{~ g / g ~ o f ~ D W ~}$ \\
\hline Bell pepper & 100 & 0.296 & 1.164 \\
& 50 & 0.156 & 1.229 \\
Correlation coefficient & 25 & 0.072 & 1.134 \\
Blackberry & 100 & 0.998 & 2.052 \\
& 50 & 0.521 & 1.908 \\
Correlation coefficient & 25 & 0.242 & 2.081 \\
Raspberry & 100 & 0.132 & \\
& 50 & 0.997 & 1.360 \\
Correlation coefficient & 25 & 0.345 & 1.338 \\
Beet & & 0.170 & 1.324 \\
& 200 & 0.084 & \\
Correlation coefficient & 100 & 1.000 & 0.141 \\
\hline
\end{tabular}

issue. Thus, the $\mathrm{IC}_{100}$, which considers a wide range of DPPH solution concentrations, is expected to offer a promising alternative that avoids the ratio issue in the DPPH assay.

\subsection{A solution for the presence of interfering pigments in the DPPH assay using a modified method}

As mentioned earlier, the co-existing pigments in extracts with absorption in the same wavelength range of DPPH radicals (around $517 \mathrm{~nm}$ ) interfere with the absorbance readings. For example, the underestimation of DPPH radical scavenging potential of extract occurs for samples containing a high concentration of pigments that can absorb at $517 \mathrm{~nm}$. This is bacause the pigments present are considered as unreacted DPPH radical in the UV-vis range (data not shown). To resolve this issue, the modified DPPH assay was employed, and its dependability and accuracy were tested by using pigment-containing extracts such as bell pepper, blackberry, raspberry, and beet (Table 3). The modified method removed the limitation of the original DPPH assay; that is, the $\mathrm{IC}_{100}$ was not influenced by the presence of pigments in the extracts, which is not possible in the original DPPH assay. The effectiveness of the modified method is easily demonstrated as the correlation coefficient between the concentrations of extracts and $\mathrm{IC}_{100}$; were 0.998 , $0.997,1.000$, and 0.993 for bell pepper, blackberry, raspberry, and beet, respectively.

Moreover, the $\mathrm{IC}_{100}$ may be calculated into a new unit in which the scavenged DPPH radical is given in $\mathrm{mg}$ (DPPH radical)/g of DW of the sample. The new unit was calculated based on the $\mathrm{IC}_{100}$ proposed here as a unit for the extracts of natural sources, which are difficult to define their molecular weight and following stoichiometry factor. The DPPH radical scavenging ability of the coloured extracts showed a nearly constant value for different concentrations of the extracts. For example, bell pepper gave values of $1.134,1.229$, and $1.164 \mathrm{DPPH}$ radical in $\mathrm{mg} / \mathrm{g}$ of DW at 25 , 50 , and $100 \mathrm{mg} / \mathrm{mL}$ concentrations of extract, respectively, show- ing a standard deviation of only 0.048 or $4.13 \%$ difference among the three tests. This indicates that the modified DPPH method effectively reduces variations caused by the presence of interfering pigments. The efficiency of the modified DPPH method for addressing the pigment issue was also proven for other extracts such as blackberry, raspberry, and beet, which showed standard deviations of $0.092,0.018$, and 0.014 , respectively. This observation confirms the suitability and feasibility of the modified DPPH assay in addressing the interference due to the co-existing pigments in the original DPPH assay. Moreover, the new unit may provide an effective way to express the antioxidant capacity of samples, as it does not require any standard curve to obtain standard compound equivalents. This reduces the required time for such determinations.

In summary, the modified DPPH assay proposed here efficiently addresses the interference by co-existing pigments in the medium which absorb in the same wavelength range. The core principle behind this achievement is that $\mathrm{IC}_{100}$, which is the required DPPH concentration to be reduced by all antioxidants in the extract, re-

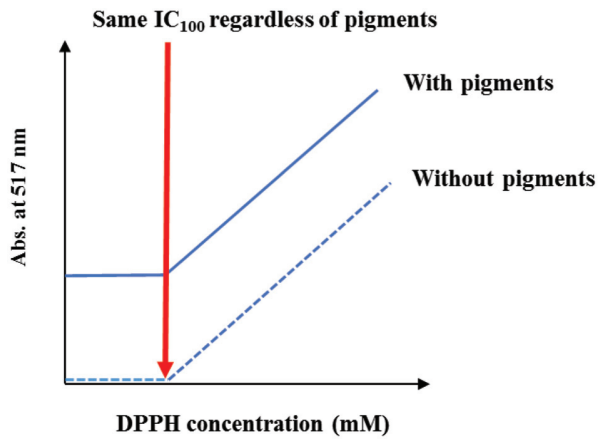

Figure 4. The $\mathrm{IC}_{100}$ of new DPPH method in the presence or absence of pigments. 
mains unchanged regardless of the presence or absence of any pigments, as depicted in Figure 4. Therefore, the modified DPPH assay also overcomes the issue associated with the colour interference caused by commonly used original DPPH assay. As already noted in the previous study we proposed the use of EPR spectroscopy to effectively address the concern about interference from colored material in the same absorption range (Yeo and Shahidi, 2019). Thus, this study will be a useful alternative for the researchers who have no access to EPR machine by employing UV-visible spectroscopy.

\section{Conclusion}

The ratio of DPPH radical to antioxidants which influences the results in the original DPPH assay was resolved by employing a modified DPPH method. The revisited method introduced $\mathrm{IC}_{100}$ which successfully eliminated/reduced the effects caused by changes in the ratio of DPPH/antioxidant. In addition, the stoichiometry factor calculated from $\mathrm{IC}_{100}$ allowed to critically discuss the possible hydrogen atom or electron donating mechanism of antioxidants by comparing the stoichiometry factor and the number of hydroxyl groups in the structure of antioxidants. Moreover, the pigment interference issue of the original DPPH assay was resolved by applying the modified DPPH assay. The modified DPPH assay might also lend itself to the exploitation of other methods when the issue of colour interference is encountered.

\section{Acknowledgments}

We are grateful to the Natural Science and Engineering Research Council (NSERC) of Canada for a Discovery Grant to FS in support of this work.

\section{References}

Ani, V., Varadaraj, M.C., and Naidu, K.A. (2006). Antioxidant and antibacterial activities of polyphenolic compounds from bitter cumin (Cuminum nigrum L.). Eur. Food Res. Technol. 224: 109-115.

Aruoma, O.I. (1998). Free radicals, oxidative stress and antioxidants in human health and disease. J. Amer. Oil Chem. Soc. 75: 199-212.

Blois, M.S. (1958). Antioxidant determinations by the use of a stable free radical. Nature 181: 1199-1200.

Bondet, V., Brand-Williams, W., and Berset, C. (1997). Kinetics and mechanisms of antioxidant activity using the DPPH radical method. LWTFood Sci. Technol. 30: 609-615.

Charrier, M.Y., Fonty, G., Gaillard-martinie, B., Ainouche, K., and Andant, G. (2006). Isolation and characterization of cultivable fermentative bacteria from the intestine of two edible snails, Helixpomatia and Cornu aspersum (Gastropoda: Pulmonata). Biol. Res. 39: 669-681.

Cheng, Z.J., Kuo, S.C., Chan, S.C., Ko, F.N., and Teng, C.M. (1998). Antioxi- dant properties of butein isolated from Dalbergia odorifera. Bioch et Bioph. Acta 1392: 291-299.

Decker, E.A. (2002). Antioxidant mechanisms. In: Akoh, C.C., and Min, D.B. (Ed.). Food lipids. Marcel Dekker, Inc., New York, pp. 520-525.

Deng, J., Cheng, W., and Yang, G. (2011). A novel antioxidant activity index (AAU) for natural products using the DPPH assay. Food Chem. 125: 1430-1435.

Elzaawely, A.A., Xuan, T.D., and Tawata, S. (2007). Essential oils, kava pyrones and phenolic compounds from leaves and rhizomes of Alpinia zerumbet (Pers.) B.L. Burtt. and R.M. Sm. and their antioxidant activity. Food Chem. 103: 486-494.

Fargere, T., Abdennadher, M., Delmas, M., and Boutevin, B. (1995). Determination of peroxides and hydroperoxides with 2,2-diphenyl-1-picrylhydrazyl (DPPH). Application to ozonized ethylene vinyl acetate copolymers (EVA). Eur. Polym. J. 31: 489-497.

Foti, M.C., and Ruberto, G. (2000). Kinetic solvent effects on phenolic antioxidants determined by spectrophotometric measurements. J. Agric. Food Chem. 49: 342-348.

Guerrero, J.L.G., Guirado, C.M., Fuentes, M.M.R., and Pérez, A.C. (2006). Nutrient composition and antioxidant activity of 10 pepper (Capsicum annuun) varieties. Eur. Food Res. Technol. 224: 1-9.

Huang, D., Ou, B., and Prior, R.L. (2005). The chemistry behind antioxidant capacity assays. J Agric. Food Chem. 53: 1841-1856.

Kanimozhi, P., and Prasad, N.R. (2009). Antioxidant potential of sesamol and its role on radiation-induced DNA damage in whole body irradiated Swiss albino mice. Env. Toxicol. Pharmacol. 28: 192-197.

Litwinienko, G., and Ingold, K.U. (2003). Abnormal effects on hydrogen atom abstractions. The reactions of phenols with 2,2-diphenyl-1-picrylhydrazyl (DPPH•) in alcohols. J. Org. Chem. 68: 3433-3438.

Madhujith, T., and Shahidi, F. (2006). Optimization of the extraction of antioxidative constituents of six barley cultivars and their antioxidant properties. J. Agric. Food Chem. 54: 8048-8057.

Pryor, W.A. (1986). Oxy-radicals and related species: their formation, lifetimes and reactions. Annu. Rev. Physiol. 48: 657-667.

Ricci, D., Fraternale, D., Giamperi, L., Bucchini, A., Epifano, F., Burini, G., and Curini, M. (2005). Chemical composition, antimicrobial and antioxidant activity of the essential oil of Teucrium marum (Lamiaceae). J. Ethnopharm. 98: 195-200.

Scherer, R., and Godoy, H.T. (2009). Antioxidant activity index (AAI) by the 2,2-diphenyl-1-picrylhydrazyl method. Food Chem. 112: 654-658.

Shahidi, F., and Peng, H. (2018). Bioaccessibility and bioavailability of phenolic compounds. J Food Bioact. 4: 11-68.

Shi, H., and Niki, E. (1998). Stoichiometric and kinetic studies on ginkgo biloba extract and related antioxidants. Lipids 33: 365-370.

Shirwaikar, A., Shirwaikar, A., Kuppusamy, R., and Punitha, I.S.R. (2006). In vitro antioxidant studies on the benzyl tetra isoquinoline alkaloid berberine. Biol. Pharmacol. Bull. 29: 1906-1910.

Sun, T., and Ho, C.T. (2005). Antioxidative activities of buck-wheat extracts. Food Chem. 90: 743-749.

van den Berg, R., Haenen, G.R.M.M., van den Berg, H., and Bast, A. (1999). Applicability of an improved trolox equivalent antioxidative capacity (TEAC) assay for evaluation of antioxidant capacity measurements of mixtures. Food Chem. 66: 511-518.

Yeo, J.D., and Shahidi, F. (2016). Review: Insoluble-bound phenolics in foods. Molecules 2016(21): 1216.

Yeo, J.D., and Shahidi, F. (2019). Critical Re-Evaluation of DPPH assay: Presence of pigments affects the results. J. Agric. Food Chem. 67: 7526-7529. 